\title{
Catalan Transform of The $k$-Lucas Numbers
}

\section{Engin ÖZKAN ${ }^{1}\left(\mathbb{D}\right.$, Merve TAŞTAN $^{2 *}$ OD Olcay GÜNGÖR ${ }^{2}$ (D)}

\author{
${ }^{1}$ Erzincan Binali Yıldırım University, Faculty of Arts and Sciences, Departments of Mathematics, 24000, \\ Erzincan, Turkey \\ ${ }^{2}$ Erzincan Binali Yıldırım University, Graduate School of Natural and Applied Sciences, Department of \\ Mathematics, 24000, Erzincan, Turkey
}

Geliş / Received: 25/10/2019, Kabul / Accepted: 21/02/2020

\begin{abstract}
Öz
Bu çalışmada, $k$-Lucas dizisinin $L_{k, n}$ Catalan dönüşümünün $C L_{k, n}$ tanımı verildi. $k$-Lucas dizisinin $L_{k, n}$ Catalan dönüşümünün $C L_{k, n}$ geren fonksiyonu elde edildi. Ayrıca, $C L_{k, n}$ dönüşümü, alt üçgen matris olan Catalan matrisi $C$ ile $n \times 1$ tipindeki $L_{k}$ matrisinin çarpımı olarak yazıldı. Hankel fonksiyonu kullanılarak $C L_{k, n}$ ler ile oluşturulan matrislerin determinantları hesaplandı.
\end{abstract}

Anahtar Kelimeler: $k$-Lucas dizisi, $k$-Fibonacci dizisi, Catalan dönüşümü, Hankel dönüşümü.

\section{Catalan Transform of The $k$-Lucas Numbers}

\begin{abstract}
In this study, the $C L_{k, n}$ description of Catalan transformation of $k$-Lucas $L_{k, n}$ sequences was given. The $C L_{k, n}$ generating function of Catalan transformation of $k$-Lucas $L_{k, n}$ sequences was obtained. And also, $C L_{k, n}$ transformation was written as the multiplying of Catalan matris $\mathrm{C}$ which is the lower triangular matris, and the $L_{k}$ matris of $n \times 1$ type. Determinants of matrices which were formed with $C L_{k, n}$ by using Hankel transform were calculated.
\end{abstract}

Keywords: $k$-Lucas sequence, $k$-Fibonacci sequence, Catalan Transform, Hankel Transform.

\section{Introduction}

For any integer $n$, it is called a generalized Fibonacci-type sequence any recurrence sequence of the following form $G(n+1)=$ $a G(n)+b G(n-1), \quad G(0)=m, G(1)=$ $t$,where $m, t, a$ and $b$ are any complex numbers. There is an extensive work in the literature concerning Fibonacci-type sequences and their applications in modern science (see e.g.[ Horadam, 1961; Bruhn, et al., 2015; Anuradha, 2008; Özkan, et al., 2018; Falcon and Plaza, 2007; Özkan and Taştan, 2019] and the references therein) The known Lucas numbers have some applications in many branches of mathematics such as group theory, calculus, applied mathematics, linear algebra, etc [Koshy, 2001; Özkan and Altun, 2019; 
Özkan, et al., 2017; Özkan, 2014; Taştan and Özkan, 2020; Özkan, et al., 2019; Özkan and Taştan, 2019].

There exist generalizations of the Lucas numbers. This paper is an extension of the work of Falcon [Falcon, 2013]. Falcon [Falcon, 2013] gave an application of the Catalan transform to the $k$-Fibonacci sequences. In this paper, we put in for Catalan transform to the $k$-Lucas sequence and present application of the Hankel transform to the Catalan transform of the $k$-Lucas sequence.

The other section of the paper is prepared as follows. The following, we introduce some fundamental definitions of $k$-Lucas numbers. In section 3, Catalan transform of $k$-Lucas sequence is given. In section 4 , generating function of Catalan transformation of $k$-Lucas sequence is obtained. Finally, we give Henkel transform of the new sequence and its determinant.

\section{2. $k$-Lucas numbers}

Let $k$ be any positive real number. Then the $k$-Lucas sequence is defined recurrently by

$$
L_{k, n+1}=k L_{k, n}+L_{k, n-1} \text { for } n \geq 1
$$

where $L_{k, 0}=2$ and $L_{k, 1}=k$. We will show the sequence with such that $\left\{L_{k . n}\right\}_{n \in N}$ from now on.

When $k=1$, the known Lucas sequence is optained.

Characteristic equation of the sequence is

$$
r^{2}-k \cdot r-1=0
$$

Its characteristic roots are

$$
r_{1}=\frac{k+\sqrt{k^{2}+4}}{2}
$$

and

$$
r_{2}=\frac{k-\sqrt{k^{2}+4}}{2}
$$

Characteristic roots verify the properties

$$
\begin{gathered}
r_{1}-r_{2}=\sqrt{k^{2}+4}=\sqrt{\Delta}=\delta \\
r_{1}+r_{2}=k \\
r_{1} \cdot r_{2}=-1
\end{gathered}
$$

Binet's formula for $L_{k, n}$ is

$$
L_{k, n}=r_{1}^{n}+r_{2}^{n} \text {. }
$$

$k$-Lucas sequence as numbered;

$$
L_{k, n+1}=k L_{k, n}+L_{k, n-1}
$$

$L_{k, 0}=2$,

$L_{k, 1}=k$,

$L_{k, 2}=k L_{k, 1}+L_{k, 0}=k^{2}+2$,

$L_{k, 3}=k L_{k, 2}+L_{k, 1}=k\left(k^{2}+2\right)+1=$ $k^{3}+3 k$,

$L_{k, 4}=k L_{k, 3}+L_{k, 2}=k\left(k^{3}+3 k\right)+k^{2}+2$,

$L_{k, 4}=k^{4}+4 k^{2}+2$,

$L_{k, 5}=k L_{k, 4}+L_{k, 3}=k\left(k^{4}+4 k^{2}+2\right)+$ $k^{3}+3 k$,

$L_{k, 5}=k^{5}+5 k^{3}+5 k$,

$L_{k, 6}=k L_{k, 5}+L_{k, 4}=k\left(k^{5}+5 k^{3}+5 k\right)+$ $k^{4}+4 k^{2}+2$,

$L_{k, 6}=k^{6}+6 k^{4}+9 k^{2}+2$, 


$$
\begin{array}{rlrl}
L_{k, 7}=k L_{k, 6}+L_{k, 5} & C L_{k, 3}=\sum_{i=1}^{3} \frac{i}{6-i}\left(\begin{array}{c}
6-i \\
3-i
\end{array}\right) L_{k, i}=k^{3}+2 k^{2}+ \\
=k\left(k^{6}+6 k^{4}+9 k^{2}+2\right)+k^{5}+5 k^{3} & 5 k+4, \\
\quad+5 k & & C L_{k, 4}=\sum_{i=1}^{4} \frac{i}{8-i}\left(\begin{array}{c}
8-i \\
4-i
\end{array}\right) L_{k, i}=k^{4}+3 k^{3}+ \\
=k^{7}+7 k^{5}+14 k^{3}+7 k . & 9 k^{2}+14 k+12,
\end{array}
$$

\subsection{Catalan Numbers}

For $n \geq 0$, the $n^{\text {th }}$ Catalan number is showed by [Barry, 2005]

$$
C_{n}=\frac{1}{n+1}\left(\begin{array}{c}
2 n \\
n
\end{array}\right) \text { or } C_{n}=\frac{(2 n) !}{(n+1) ! n !}
$$

$C L_{k, 5}=\sum_{i=1}^{5} \frac{i}{10-i}\left(\begin{array}{c}10-i \\ 5-i\end{array}\right) L_{k, i}=k^{5}+$

$4 k^{4}+14 k^{3}+30 k^{2}+46 k+36$,

and its generating function is given by

$$
c(x)=\frac{1-\sqrt{1-4 x}}{2 x} .
$$

For some $n$, the first Catalan numbers are

$$
C L_{k, 6}=\sum_{i=1}^{6} \frac{i}{12-i}\left(\begin{array}{c}
12-i \\
6-i
\end{array}\right) L_{k, i}=k^{6}+
$$$$
5 k^{5}+20 k^{4}+53 k^{3}+107 k^{2}+151 k+
$$

114

$C L_{k, 7}=\sum_{i=1}^{7} \frac{i}{14-i}\left(\begin{array}{c}14-i \\ 7-i\end{array}\right) L_{k, i}=k^{7}+$

$\{1,1,2,5,14,132,429,1430,4862,16796, \ldots\} 509 k+372$.

,from now on OEIS, as A000108 in http://en.wikipedia.org/wiki/Catalan number.

\section{Catalan transform of the $C$ as}

\section{$\boldsymbol{k}$-Lucas sequence}

Following [Barry, 2005], we define the Catalan transform of the $k$-Lucas sequence $\left\{L_{k, n}\right\}$ as

$$
C L_{k, n}=\sum_{i=1}^{n} \frac{i}{2 n-i}\left(\begin{array}{c}
2 n-i \\
n-i
\end{array}\right) L_{k, i} \text { for } n \geq 1
$$

with $C L_{k, 0}=0$.

We can give some members of Catalan transform of the first $k$-Lucas numbers.

These are the polynomials in $k$ :

$C L_{k, 1}=\sum_{i=1}^{1} \frac{i}{2-i}\left(\begin{array}{c}2-i \\ 1-i\end{array}\right) L_{k, i}=k$,

$C L_{k, 2}=\sum_{i=1}^{2} \frac{i}{4-i}\left(\begin{array}{c}4-i \\ 2-i\end{array}\right) L_{k, i}=k^{2}+k+2, \quad$ where

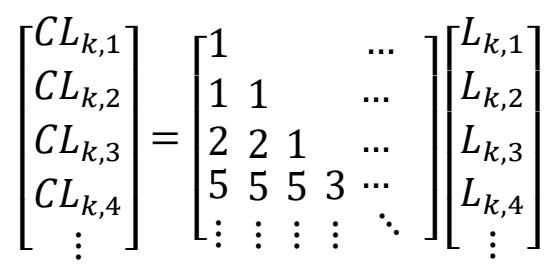

$$
\begin{aligned}
& {\left[\begin{array}{c}
k \\
k^{2}+k+2 \\
k^{3}+2 k^{2}+5 k+4 \\
k^{4}+3 k^{3}+9 k^{2}+14 k+12 \\
\vdots
\end{array}\right]} \\
& =\left[\begin{array}{ccccc}
1 & & & \cdots \\
1 & 1 & & \cdots \\
2 & 2 & 1 & \cdots \\
5 & 5 & 5 & 3 & \cdots \\
\vdots & \vdots & \vdots & \vdots & \ddots
\end{array}\right]\left[\begin{array}{c}
k \\
k^{2}+2 \\
k^{3}+3 k \\
k^{4}+4 k^{2}+2 \\
\vdots
\end{array}\right]
\end{aligned}
$$




$$
C_{i, j}=\sum_{r=j-1}^{i-1} C_{i-1, r}
$$

The lower triangular matrix $C_{n, n-i}$ is known as the Catalan triangle and its elements verify the formula

$$
C_{n, n-i}=\frac{(2 n-i) !(i+1)}{(n-i) !(n+1) !}
$$

with $0 \leq i \leq n$.

\section{Generating function}

We know that the generating function of the $k$-Lucas polynomials and the generating function of the Catalan numbers, respectively, are $L_{k}(x)=\frac{2-k x}{1-k x-x^{2}} \quad$ and $c(x)=\frac{1-\sqrt{1-4 x}}{2 x}$ in $[1,14]$.

Let $C(x)$ and $A(x)$, respectively, be the generating function of the sequence of the Catalan numbers $\left\{C_{n}\right\}$ and and the generating function of the sequence $\left\{a_{n}\right\}$. It is proved that $A(x * c(x))$ is the generating function of the Catalan transform of this last sequence [Barry, 2005]. Consequently, we obtain that the generating function of the Catalan transform of the $k$-Lucas sequence $\left\{L_{k, n}\right\}$ is

$$
\begin{aligned}
& C L_{k}(x)=L_{k}(x * C(x)) \\
& =\frac{4-k+k \cdot \sqrt{1-4 x}}{1+2 x-k+(k+1) \sqrt{1-4 x}} .
\end{aligned}
$$

\section{Hankel Transform}

$A=\left\{a_{0}, a_{1}, a_{2}, \ldots,\right\}$ is a sequence of real numbers [Cvetkovi'c, et al., 2002; Layman, 2001]. The Hankel transform of the sequence $A$ is the sequence of determinants $H_{n}=$ $\operatorname{Det}\left[a_{i+j-2}\right]$, i.e.,

$$
H_{n}=\left|\begin{array}{ccccc}
a_{0} & a_{1} & a_{2} & a_{3} & \ldots \\
a_{1} & a_{2} & a_{3} & a_{4} & \ldots \\
a_{2} & a_{3} & a_{4} & a_{5} & \ldots \\
a_{3} & a_{4} & a_{5} & a_{6} & \ldots \\
. & . & . & . & . \\
. & . & . & . & . \\
. & . & . & . & .
\end{array}\right| .
$$

The upper-left $n \times n$ subdeterminant of $H_{n}$ is called the Hankel determinant of order $n$ of the sequence $A$.

The Hankel transform of the Catalan sequence is the sequence $\{1,1,1, \ldots\}$ [Sloane, 2007] and the Hankel transform of the sum of consecutive generalized Catalan numbers is the bisection of classical Fibonacci sequence [Rajkovi'c, et al.,2007]. It is very interesting the study of the Catalan transform of this $k$-Lucas sequence, as we will see in the sequel.

Considering the Catalan transform of the $k$-Lucas sequence of the preceding subsection, we find out:

$$
H C L_{1}=\operatorname{Det}[k]=k
$$

$$
\begin{gathered}
H C L_{2}=\left|\begin{array}{cc}
k & k^{2}+k+2 \\
k^{2}+k+2 & k^{3}+2 k^{2}+5 k+4
\end{array}\right| \\
=-4
\end{gathered}
$$

$$
\begin{aligned}
& H_{C L} L_{3} \\
& =\left|\begin{array}{ccc}
k & k^{2}+k+2 & k^{3}+2 k^{2}+5 k+4 \\
k^{2}+k+2 & k^{3}+2 k^{2}+5 k+4 & k^{4}+3 k^{3}+9 k^{2}+14 k+12 \\
k^{3}+2 k^{2}+5 k+4 & k^{4}+3 k^{3}+9 k^{2}+14 k+12 & k^{5}+4 k^{4}+14 k^{3}+30 k^{2}+46 k+36
\end{array}\right| \\
& =k^{3}-4 k^{2}-8 k-16 .
\end{aligned}
$$

\section{References}

Barry, P. 2005. "A Catalan transform and related transformations on integer sequences', J.Integer Seq., 8(4), 1-24.

Cvetkovi'c, A., Rajkovi'c, R., and Ivkovi'c, M. 2002. 'Catalan numbers, and Hankel 
transform, and Fibonacci numbers" J. http://en.wikipedia.org/wiki/Catalan number. Integer Seq. 5(1), 1-8.

Layman, J. W. 2001. 'The Hankel transform and some of its properties', J. Integer Seq. $4(1), 1-11$.

Rajkovi'c, P. M., Petkovi'c, M. D. and Barry, P. 2007. "The Hankel transform of the sum of consecutive generalized Catalan numbers. Integral Transforms Spec. Funct'. 18(4), 285-296.

Horadam, A. F. 1961. "A generalized Fibonacci sequence''. Amer. Math. Monthly, 68 (5), 455-459.

Bruhn, H. Gellert, L. Günther, J. 2015. 'Jacobsthal numbers in generalized Petersen graphs" Electronic Notes in Discrete Mathematics, 49, 465-472.

Anuradha, N. 2008. 'Number of points on certain hyperelliptic curves defined over finite fields" Finite Fields and Their Applications, 14, 314-328.

Falcon, S. (2013). 'Catalan Transform of the $k$-Fibonacci sequence". Commun. Korean Math. Soc. 28(4), 827-832.

Özkan, E., Taştan, M., Aydoğdu, A. 2018. '2-Fibonacci Polynomials in the Family of Fibonacci Numbers", Notes on Number Theory and Discrete Mathematics, 24, 47-55.

Koshy, T. 2001. 'Fibonacci and Lucas Numbers with Applications". New York, NY: Wiley-Interscience Publication.

Falc'on, S., and Plaza, A. 2007. 'On the Fibonacci k-numbers", Chaos Solitons Fractals 32(5), 1615-1624.

Sloane, N. J. A., 2007. The on-line encyclopedia of integer sequences, www.research.att.com/njas/sequences/

Boussayoud, A., Kerada, M. and , Harrouche, N. 2017. "On the $\mathrm{k}$-Lucas Numbers and Lucas Polynomials", Turkish Journal of Analysis and Number Theory, 5(4), 121-125.

Özkan E., Taştan M. 2019."On Gauss Fibonacci Polynomials, Gauss Lucas Polynomials and Their Applications ", Communications in Algebra, https://doi.org/10.1080/00927872.2019.1670 193

Özkan E., Altun İ. 2019. "Generalized Lucas Polynomials And Relationships Between The Fibonacci Polynomials And Lucas Polynomials ", Communications in Algebra, 47, 10-12.

Özkan E., Altun İ., Göçer A. 2017, "On Relationship Among A New Family of kFibonacci, k-Lucas Numbers, Fibonacci And Lucas Numbers", Chiang Mai Journal of Sc1ence, 44, 1744-1750.

Özkan E. 2014. "Truncated Lucas sequence and its period", Applied Mathematics and Computation, 232, 285-291.

Taştan, M., Özkan, E. 2020. 'Catalan Transform of The k-Jacobsthal Sequence', Electronic Journal of Mathematical Analysis and Applications, 8(2), 70-74.

Özkan, E., Taştan, M., Aydoğdu, A. 2019. '3-Fibonacci Polynomials in The Family of Fibonacci Numbers', Erzincan University Journal of the Institute of Science and Technology, 12(2), 926-933.

Özkan, E., Taştan, M. 2019. ' $k$-Fibonacci Polynomials in The Family of Fibonacci Numbers", Research \& Reviews: Discrete Mathematical Structure, 6(3),19-22. 